\title{
Quantitative Imaging of Fluorescent Protein FRET
}

\author{
D.W. Piston, M.A Rizzo, G. Springer, K. Segawa \\ Department of Molecular Physiology and Biophysics, Vanderbilt University, Nashville, TN 37232
}

The convergence of newly developed instrumentation and optical probes is allowing us to examine quantitatively dynamic processes within living cells, using different color mutants of the Green Fluorescent Protein (GFP). A key methodology in this examination is Förster resonance energy transfer (FRET). The most popular pair of fluorescent proteins for FRET experiments is cyan (CFP) and yellow (YFP), and the preferred versions of these variants are the CFP Cerulean [1] and the YFP Venus [2]. We will concentrate on the methods and limitations of those methods for the assay of FRET between Cerulean and Venus.

One of the most robust applications of GFP-based FRET is in the creation of intramolecular biosensors where both the donor (CFP) and the acceptor (YFP) are attached to the same molecule. This constrains the distance between the two fluorescent proteins and allows the assay of changes in the distance or orientation between them. We have used this approach to create biosensors for various biological processes. Among these, one stands out in terms of new biological insight and that is the sensor for glucokinase (GK) activity in pancreatic beta cells [3]. GK is the glucose sensing enzyme of the beta cell and is the rate-limiting step in insulin secretion. By fusing two different colored GFPs (CFP on one end of GK and YFP on the other, we have discovered that the location and activity of $\beta$ cell GK is acutely regulated by both glucose and insulin. In addition, the changes in the FRET signal between the CFP and YFP revealed that the changes in location correlate with increased GK activity. Thus, the FRET is sensitive to conformational changes in the GK molecule that occur during its regulation. Using this GK biosensor, we found that glucoseinduced changes in FRET require autostimulation of $\beta$ cells by secreted insulin. Further, this stimulation is dependent on GK interaction with a protein complex including nitric oxide synthase (NOS).

To effectively exploit this biosensor, we utilized a number of approaches for FRET quantification. Spectral imaging has been crucial in allowing us to measure quantitatively FRET efficiencies between both CFP/YFP pairs as well and GFP/YFP pairs. In this approach, a single excitation wavelength is used, but the entire fluorescence spectrum is collected. With a spectral resolution of $10 \mathrm{~nm}$, it is straight forward to quantitatively unmix the CFP and YFP fluorescence spectra, which in turn permits determination of the number of CFP and YFP molecules, as well as the amount of FRET between them. Spectral imaging is simple given the proper instrumentation (available from many vendors), and yields reliable numbers. Another approach has been the use of fluorescence anisotropy. Since FPs are large and rotate only a small amount during their nanosecond excited-state lifetime, their fluorescence anisotropy is high $(\sim 0.3)$ in the absence of FRET. However, FRET can dramatically reduce the anisotropy to levels below 0.1 [4]. While the anisotropy approach is difficult to quantify, it is ideal for screening purposes, where the desired readout is FRET or no FRET. We have found that lifetime imaging provides the most reliable numbers for FRET efficiency, but even with commercial systems, these measurements are difficult to obtain, analyze and interpret. Finally, we have used the most common method: acceptor photobleaching, which is also called donor dequenching. While this method is simple, we do not find that the results it produces agree with those obtained by spectral or lifetime imaging. There are two reasons that donor dequenching works poorly for CFP/YFP FRET quantification. First, the laser line that is typically used is the $458 \mathrm{~nm}$ argon line, and this generates considerable direct 
excitation of the YFP (see Fig. 1). Secondly, the YFP absorption of the laser can cause an inner filter effect that reduces the CFP excitation. Upon YFP photobleaching, then, not only is the FRET signal eliminated, but increased CFP excitation is also generated. For our CFP/YFP FRET standards that we know have 20\% FRET efficiency, the acceptor photobleaching method with a $458 \mathrm{~nm}$ excitation yields a result of $30 \%$ FRET efficiency. While this acceptor photbleaching approach can still be used rigorous establish that there is no FRET present, it is prone to an artifact that might lead to an over estimation of the FRET present, or even to false positive signals.

\section{$\underline{\text { References }}$}

[1] Rizzo, M.A., G.H. Springer, B. Granada, D.W. Piston. Nat. Biotechol. 22:445-449 (2004).

[2] Nagai, T., et al. Nat. Biotechnol. 20:87-90 (2002).

[3] Rizzo, M.A., D.W. Piston. J. Cell Biol. 161:243 (2003).

[4] Rizzo, M.A., D.W. Piston. Biophys. J. 88:L14-L16 (2005).

[5] This research was supported by NIH grants DK53434, GM72048, DK60275, and DK59737.
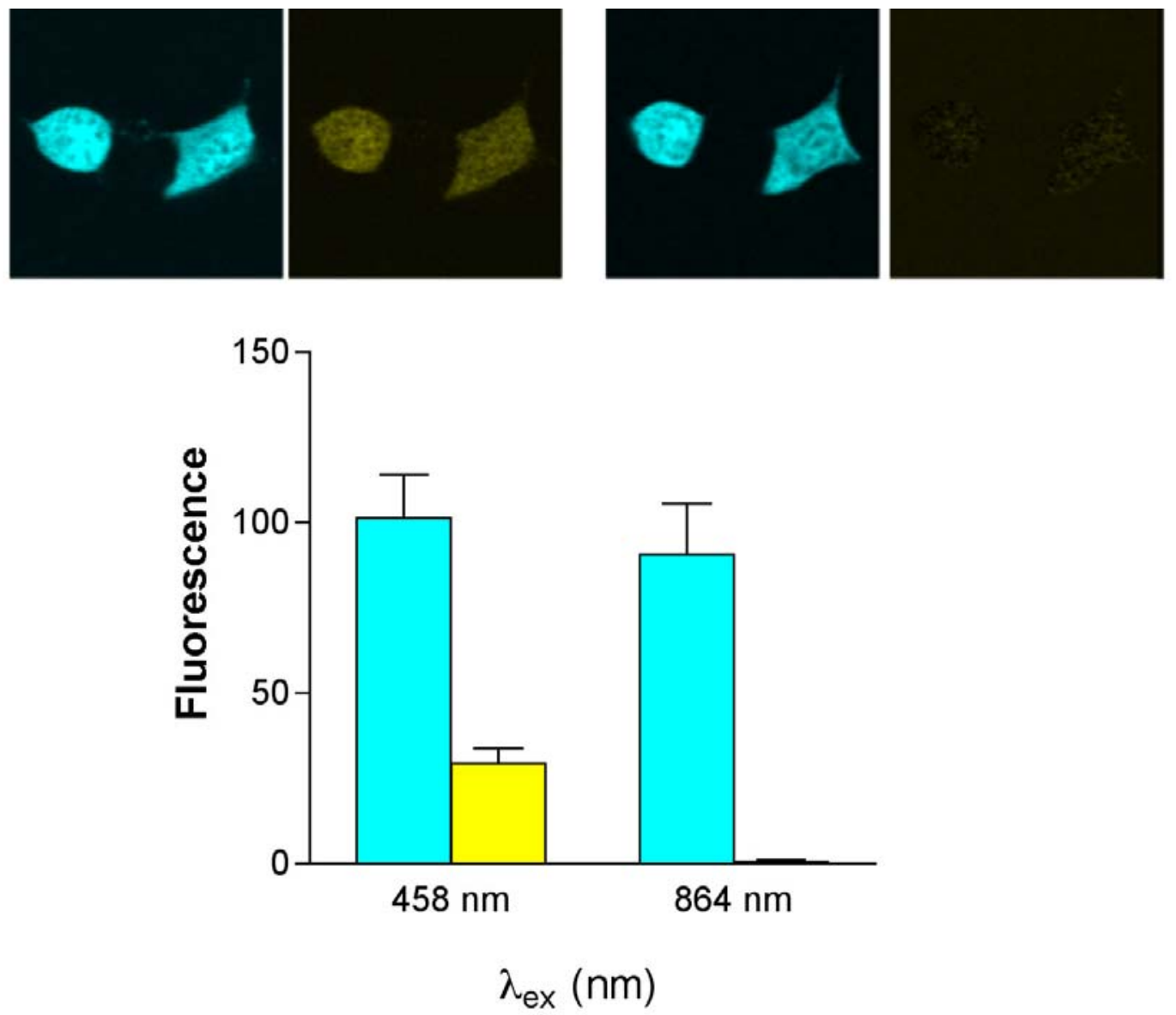

FIG. 1. CFP-YFP co-expression (no FRET) shows considerable signal in the "FRET" channel when excited at $458 \mathrm{~nm}$. This is due to direct excitation of the YFP acceptor. Excitation with two-photon excitation at $864 \mathrm{~nm}$ greatly reduces this direct absorbance by the acceptor. Similarly, use of $<440$ $\mathrm{nm}$ excitation would alleviate the problem in one-photon confocal microscopy, but most installed confocal microscopes do not offer that option. 